\title{
CURVATURE ESTIMATES FOR COMPLETE AND BOUNDED SUBMANIFOLDS IN A RIEMANNIAN MANIFOLD
}

\author{
YOSHIHISA KITAGAWA
}

\begin{abstract}
Let $M$ be a complete $n$-dimensional submanifold in the $(2 n-1)$ dimensional Euclidean space, with scalar curvature bounded from below. Baikousis and Koufogiorgos proved that the sectional curvature of $M$ satisfies sup $K_{M}>\lambda^{-2}$ if $M$ is contained in a ball of radius $\lambda$. We extend this result to the case that the ambient space is a complete simply connected Riemannian manifold of nonpositive curvature.
\end{abstract}

1. Introduction. For $p<n$, let $M$ be a complete $n$-dimensional Riemannian submanifold in the $(n+p)$-dimensional Euclidean space $E^{n+p}$. Under the assumption that the scalar curvature of $M$ has a lower bound, Baikousis and Koufogiorgos [1] proved that if $M$ is contained in a ball of radius $\lambda$, then the sectional curvature $K_{M}$ of $M$ satisfies sup $K_{M} \geqslant \lambda^{-2}$. In this note we obtain a natural extension of the above inequality when the ambient space is a complete simply connected $(n+p)$ dimensional Riemannian manifold of nonpositive curvature. To state our result, we introduce a continuous function $f:[0, \infty) \rightarrow[1, \infty)$ by

$$
f(t)=\left\{\begin{array}{l}
1 \quad \text { if } t=0, \\
t \operatorname{coth}(t) \quad \text { if } t>0 .
\end{array}\right.
$$

THEOREM. For $p<n$, let $M$ be a complete $n$-dimensional Riemannian submanifold in $a(n+p)$-dimensional complete simply connected Riemannian manifold $\bar{M}$ whose sectional curvature satisfies $a \leqslant K_{\bar{M}} \leqslant b \leqslant 0$. If $M$ is contained in a geodesic ball of radius $\lambda$ and the scalar curvature of $M$ has $a$ lower bound, then the sectional curvature $K_{M}$ of $M$ satisfies $\sup K_{M}>a+\lambda^{-2}\{f(\sqrt{-b} \lambda)\}^{2}$.

The author sincerely thanks Professor S. Tanno for valuable suggestions.

2. Proof of Theorem. We denote the Riemannian metric on $\bar{M}$ (resp. $M$ ) by $\langle$, $\rangle$ (resp. $\langle$,$\rangle ), the Riemannian connection by \bar{\nabla}$ (resp. $\nabla$ ), the Riemannian curvature tensor by $\bar{R}$ (resp. $R$ ) and the second fundamental form with respect to the immersion $M \subset \bar{M}$ by $\alpha$.

Since the scalar curvature of $M$ has a lower bound, we may assume inf $K_{M}>$ $-\infty$. Let $d$ be the distance function on $\bar{M}$ and choose a point $\bar{o} \in \bar{M}$ such that $d(\bar{o}, x) \leqslant \lambda$ for all $x \in M$. We define a smooth function $F: M \rightarrow R$ by $F(x)=$ $\{d(\bar{o}, x)\}^{2} / 2$. Then by $\left[4\right.$, Theorem $\left.\mathrm{A}^{\prime}\right]$ there exists a sequence $\left\{x_{k}\right\}_{k=1}^{\infty}$ in $M$ such that

$$
\left\|\operatorname{grad} F\left(x_{k}\right)\right\|<k^{-1} \text {, }
$$

Received by the editors December 4, 1980.

1980 Mathematics Subject Classification. Primary 53C40; Secondary 53C20.

(c) 1981 American Mathematical Society 0002-9939/81/0000-0531/\$01.75 


$$
\begin{gathered}
\nabla^{2} F(X, X)<k^{-1} \quad \text { for all unit vectors } X \in T_{x_{k}} M, \\
\qquad \lim _{k \rightarrow \infty} F\left(x_{k}\right)=\sup F,
\end{gathered}
$$

where $\nabla^{2} F$ denotes the Hessian of $F$ with respect to the Riemannian metric on $M$.

LEMMA 1. Let $\gamma:[0,1] \rightarrow \bar{M}$ be a geodesic in $\bar{M}$ such that $\gamma(0)=\bar{o}$ and $\gamma(1) \in M$. Then

$$
\begin{aligned}
\nabla^{2} F(X, X) \geqslant & \langle\alpha(X, X), \dot{\gamma}(1)\rangle+L^{-2}\langle X, \dot{\gamma}(1)\rangle^{2} \\
& +\left(\|X\|^{2}-L^{-2}\langle X, \dot{\gamma}(1)\rangle^{2}\right) f(\sqrt{-b} L),
\end{aligned}
$$

for all vectors $X$ tangent to $M$ at $\gamma(1)$, where $L$ is the length of $\gamma$.

Proof. Let $c(s)$ be the geodesic in $M$ such that $\dot{c}(0)=X$ and let $\gamma_{s}:[0,1] \rightarrow \bar{M}$ be the geodesic such that $\gamma_{s}(0)=\overline{0}$ and $\gamma_{s}(1)=c(s)$. Then we have $\nabla^{2} F(X, X)=$ $\left.F(c(s))^{\prime \prime}\right|_{s=0}=\left.E\left(\gamma_{s}\right)^{\prime \prime}\right|_{s=0}$, where $E\left(\gamma_{s}\right)$ is the energy of $\gamma_{s}$ defined by $E\left(\gamma_{s}\right)=$ $\int_{0}^{1}\left\langle\dot{\gamma}_{s}, \dot{\gamma}_{s}\right\rangle / 2$. Let $V$ be the variation vector field along $\gamma$ with respect to the variation $\left\{\gamma_{s}\right\}$. Then a calculation shows that

$$
\left.E\left(\gamma_{s}\right)^{\prime \prime}\right|_{s=0}=\langle\alpha(X, X), \dot{\gamma}(1)\rangle+I(V, V),
$$

where $I(V, V)=\int_{0}^{1}\left\langle\left\langle\bar{\nabla}_{\dot{\gamma}} V, \bar{\nabla}_{\dot{\gamma}} V\right\rangle+\langle\bar{R}(\dot{\gamma}, V) \dot{\gamma}, V\rangle\right\}$. Let $\tilde{M}$ be the $(\tilde{n}+p)-$ dimensional space form with constant curvature $b$ and let $\sigma:[0,1] \rightarrow \tilde{M}$ be a geodesic with length $L$. We construct a vector field $W$ along $\sigma$ such that $\|V\|=$ $\|W\|,\left\|\bar{\nabla}_{\dot{\gamma}} V\right\|=\left\|\tilde{\nabla}_{\dot{\sigma}} W\right\|$ and $\langle V, \dot{\gamma}\rangle=\langle W, \dot{\sigma}\rangle$, where $\tilde{\nabla}$ is the Riemannian connection with respect to the Riemannian metric $\langle$, $\rangle$ on $\tilde{M}$. Then $K_{\bar{M}}<b$ implies $I(V, V) \geqslant I(W, W)$. Let $J$ be the Jacobi field along $\sigma$ determined by $J(0)=0$ and $J(1)=W(1)$. Then [2, First lemma, p. 24] implies $I(W, W)>I(J, J)$. Let $U$ be the parallel vector field along $\sigma$ determined by $U(1)=J(1)-L^{-2}\langle J(1), \dot{\sigma}(1)\rangle \dot{\sigma}(1)$, and let $g:[0,1] \rightarrow R$ be the solution of $g^{\prime \prime}+b L^{2} g=0$ determined by $g(0)=0$ and $g(1)=1$. Then we have $J(t)=g(t) U(t)+\left\{L^{-2}\langle J(1), \dot{\sigma}(1)\rangle t\right\} \dot{\sigma}(t)$ and $g^{\prime}(1)=f(\sqrt{-b} L)$. Hence we see that $I(J, J)=\left.\left\langle\tilde{\nabla}_{\dot{j}} J, J\right\rangle\right|_{t-1}=g^{\prime}(1)\|U(1)\|^{2}+$ $L^{-2}\langle J(1), \dot{\sigma}(1)\rangle^{2}=f(\sqrt{-b} L)\left(\|X\|^{2}-L^{-2}\langle X, \dot{\gamma}(1)\rangle^{2}\right)+L^{-2}\langle X, \dot{\gamma}(1)\rangle^{2}$. Q.E.D.

Let $\gamma_{k}:[0,1] \rightarrow \bar{M}$ be the geodesic such that $\gamma_{k}(0)=\bar{o}$ and $\gamma_{k}(1)=x_{k}$, and let $\lambda_{k}$ be the length of $\gamma_{k}$. We set $\lambda_{\infty}=\sup \{d(\bar{o}, x) \mid x \in M\}$, then (4) implies $\lim _{k \rightarrow \infty} \lambda_{k}$ $=\lambda_{\infty}>0$. Therefore we may assume $\lambda_{k}>0$ for all $k$. Let $X$ be a unit vector in $T_{x_{k}} M$. Then by (3) and Lemma 1 we have

$$
\left.k^{-1}\right\rangle\left\langle\alpha(X, X), \dot{\gamma}_{k}(1)\right\rangle-\lambda_{k}^{-2}\left\langle X, \dot{\gamma}_{k}(1)\right\rangle^{2}\left\{f\left(\sqrt{-b} \lambda_{k}\right)-1\right\}+f\left(\sqrt{-b} \lambda_{k}\right) .
$$

Since $\left\langle X, \dot{\gamma}_{k}(1)\right\rangle=\left\langle X, \operatorname{grad} F\left(x_{k}\right)\right\rangle$, (2) implies $\left\langle X, \dot{\gamma}_{k}(1)\right\rangle^{2}\left\langle k^{-2}\right.$. Hence we have

$$
\|\alpha(X, X)\|>\left\{f\left(\sqrt{-b} \lambda_{k}\right)-A_{k}\right\} / \lambda_{k}
$$

for all unit vectors $X \in T_{x_{k}} M$, where $A_{k}=k^{-1}+k^{-2} \lambda_{k}^{-2}\left\{f\left(\sqrt{-b} \lambda_{k}\right)-1\right\}$. Since $\lim _{k \rightarrow \infty}\left\{f\left(\sqrt{-b} \lambda_{k}\right)-A_{k}\right\}=f\left(\sqrt{-b} \lambda_{\infty}\right)>1$, we may assume $f\left(\sqrt{-b} \lambda_{k}\right)-A_{k}>0$ for all $k$. Hence (5) implies $\alpha(X, X) \neq 0$ for all nonzero vectors $X \in T_{x_{k}} M$. Now we recall the following lemma [3, p. 28].

Lemma 2. Let $\alpha: R^{n} \times R^{n} \rightarrow R^{p}$ be symmetric bilinear and satisfy $\alpha(X, X) \neq 0$ for all nonzero $X \in R^{n}$. If $p<n$, there exist linearly independent vectors $X, Y \in R^{n}$ such that $\alpha(X, Y)=0, \alpha(X, X)=\alpha(Y, Y)$. 
By Lemma 2 there exist linearly independent vectors $X_{k}, Y_{k}$ in $T_{x_{k}} M$ such that $\alpha\left(X_{k}, Y_{k}\right)=0, \alpha\left(X_{k}, X_{k}\right)=\alpha\left(Y_{k}, Y_{k}\right)$. Hence by the Gauss equation, we have $\left\langle R\left(X_{k}, Y_{k}\right) Y_{k}, X_{k}\right\rangle=\left\langle\bar{R}\left(X_{k}, Y_{k}\right) Y_{k}, X_{k}\right\rangle+\left\|\alpha\left(X_{k}, X_{k}\right)\right\| \cdot\left\|\alpha\left(Y_{k}, Y_{k}\right)\right\|$. Let $\bar{K}\left(X_{k}, Y_{k}\right)$ (resp. $\left.K\left(X_{k}, Y_{k}\right)\right)$ be the sectional curvature of $\bar{M}$ (resp. $M$ ) for the plane spanned by $X_{k}$ and $Y_{k}$. Then by (5) we see that

$$
\begin{aligned}
K\left(X_{k}, Y_{k}\right)= & \bar{K}\left(X_{k}, Y_{k}\right)+\left\|\alpha\left(X_{k}, X_{k}\right)\right\| \\
& \cdot\left\|\alpha\left(Y_{k}, Y_{k}\right)\right\|\left(\left\|X_{k}\right\|^{2}\left\|Y_{k}\right\|^{2}-\left\langle X_{k}, Y_{k}\right\rangle^{2}\right)^{-1} \\
\geqslant & a+\left\|\alpha\left(X_{k}, X_{k}\right)\right\| \cdot\left\|\alpha\left(Y_{k}, Y_{k}\right)\right\| \cdot\left\|X_{k}\right\|^{-2}\left\|Y_{k}\right\|^{-2} \\
> & a+\lambda_{k}^{-2}\left\{f\left(\sqrt{-b} \lambda_{k}\right)-A_{k}\right\}^{2} .
\end{aligned}
$$

Letting $k$ go to infinity, we have $\sup K_{M}>a+\lambda_{\infty}^{-2}\left\{f\left(\sqrt{-b} \lambda_{\infty}\right)\right\}^{2}$. Since $\lambda_{\infty}<\lambda$ and the function $t \mapsto t^{-2}\{f(\sqrt{-b} t)\}^{2}$ is decreasing, we have sup $K_{M} \geqslant a+$ $\lambda^{-2}\{f(\sqrt{-b} \lambda)\}^{2}$. This completes the proof of the theorem.

\section{REFERENCES}

1. C. Baikousis and T. Koufogiorgos, Isometric immersions of complete Riemannian manifolds into Euclidean space, Proc. Amer. Math. Soc. 79 (1980), 87-88.

2. J. Cheeger and D. Ebin, Comparison theorems in Riemannian geometry, North-Holland, Amsterdam, 1975.

3. S. Kobayashi and K. Nomizu, Foundation of differential geometry, Vol. II, Interscience, New York, 1967.

4. H. Omori, Isometric immersions of Riemannian manifolds, J. Math. Soc. Japan 19 (1967), 205-214.

Mathematical Institute, Tôhoku University, SENDaI, 980 JaPan 